\title{
HI Absorption as a Tracer of Nuclear Rings in Galaxies
}

\author{
Bärbel Koribalski \\ Australia Telescope National Facility (CSIRO), P. 0.Box 76, Epping, \\ NSW 2121, Australia
}

\begin{abstract}
H I absorption measurements against strong continuum background sources are an excellent tool to study the cold ISM in the line of sight to the object (e.g. pulsars, galaxies, quasars). Here we present a brief overview of such studies against the nuclear region of starburst galaxies. The high angular resolution provided by synthesis telescopes allows us to resolve the central region of nearby galaxies and thus investigate the gas kinematics close to the nucleus. The detection of fast-rotating nuclear rings is related to the bars in these galaxies which in turn might have been triggered by tidal galaxy-galaxy interactions.
\end{abstract}

\section{Introduction}

What appears to be a 'hole' or 'depression' in the central H I gas distribution of galaxies (see Figure 1-4) can often be attributed to absorption of cold neutral hydrogen gas against strong $20-\mathrm{cm}$ continuum emission. In starburst galaxies, which have very bright central regions due to the mostly non-thermal emission from supernovae remnants, this seems to be a rather common effect. While the $\mathrm{H}$ I emission provides us with the overall gas dynamics in a galaxy, the absorption can be used to trace the nuclear gas kinematics. In addition, both together can be used to calculate the spin temperature of the gas (see e.g. Dickey, Brinks, \& Puche 1992). Several galaxies (e.g. NGC 1808, see below) show H I absorption lines over about the same velocity range as the $\mathrm{H}$ I emission while both originate from completely different regions within the galaxy. In those cases where the continuum emission has been resolved, the absorption lines indicate fast rotation of the gas in the nuclear region. The galaxies with broad $\mathrm{H}$ I absorption lines can be divided into two groups: 1) nearby barred starburst galaxies, many of which also have active nuclei and megamasers (a few examples are discussed below), 2) distant merging or strongly interacting galaxies (see Koribalski 1993). In the following we mainly concentrate on the first group of galaxies. The nuclear rings, which are often also visible at other wavelengths, are therefore most likely related to the inner Lindblad resonance(s) created by the kinematics of the bar (see e.g. Combes 1988).

\section{The Nearby Starburst Galaxy NGC 253}

NGC 253 is a rather close $(D=3.4 \mathrm{Mpc})$ edge-on galaxy $\left(i \sim 78^{\circ}\right)$ and the brightest member of the Sculptor group. Its nuclear region is particularly active, 

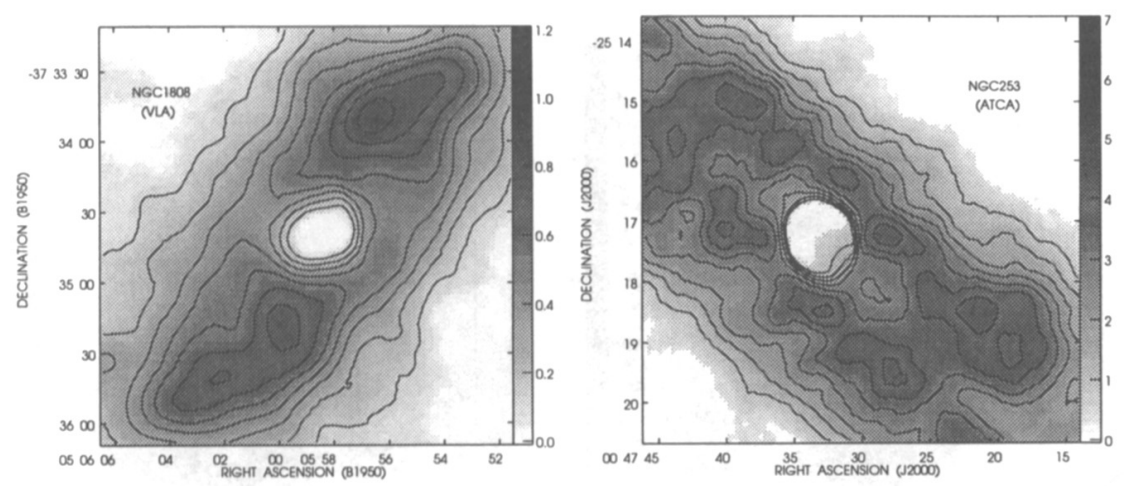

Figure 1. $\mathrm{H}$ I distribution in the disks of the starburst galaxies NGC 1808 and NGC 253. The central 'hole' is caused by $\mathrm{H}$ I absorption against the nuclear continuum emission. The data were obtained with the Very Large Array (VLA) and the Australia Telescope Compact Array (ATCA), respectively.

revealing numerous compact radio sources (Antonucci \& Ulvestad 1988), as well as bright optical (Pence 1981) and infrared emission lines (Forbes et al. 1993). A 2-kpc bar has been found in the near-infrared (Scoville et al. 1985, Forbes \& DePoy 1992) and optical (Pence 1980). High-resolution H I measurements have been carried out by Koribalski, Whiteoak, \& Houghton (1995) with the ATCA (see Figure 1). The $\mathrm{H} \mathrm{I}$ emission covers a velocity range from $\sim 6$ to $468 \mathrm{~km} \mathrm{~s}^{-1}$, significantly larger than the $\sim 52$ to $356 \mathrm{~km} \mathrm{~s}^{-1}$ covered by the absorption. A preliminary analysis of the nuclear velocity field as obtained from the $\mathrm{H}$ I absorption indicates a fast-rotating ring of cold gas as well as outflow of gas (see also Combes, Gottesman, \& Weliachew 1977; Dickey, Brinks, \& Puche 1992). The nuclear ring, which has also been inferred from $\mathrm{H} \alpha$ observations (Arnaboldi et al. 1995), is most likely situated at the inner Lindblad resonance(s).

\section{The Peculiar Starburst Galaxy NGC 1808}

The southern galaxy NGC 1808 is a nearby $(D=10.9 \mathrm{Mpc})$ example of an extraordinary type of spiral with nuclear starburst activity $\left(L_{\mathrm{FIR}} \approx 2 \times 10^{10}\right.$ $\left.L_{\odot}\right)$; for a comprehensive overview see Koribalski (1993). VLA measurements of its nuclear region reveal that the broad $\left(\Delta v \approx 360 \mathrm{~km} \mathrm{~s}^{-1}\right) \mathrm{H} \mathrm{I}$ absorption line seen at low angular resolution is in fact a much narrower line which shifts its center velocity over the face of the continuum (Koribalski, Dickey, \& Mebold 1993). This is interpreted as a torus of cold, dense gas with a rotation velocity of $\sim 250 \mathrm{~km} \mathrm{~s}^{-1}$ and radius $500 \mathrm{pc}$. A stellar bar (length $\sim 3^{\prime}$ or $6 \mathrm{kpc}$ ), which was discovered in the $\mathrm{H} \alpha$ line (Phillips 1993, Koribalski \& Dettmar 1993), might be causing the gas to accumulate at the inner Lindblad resonance(s). 

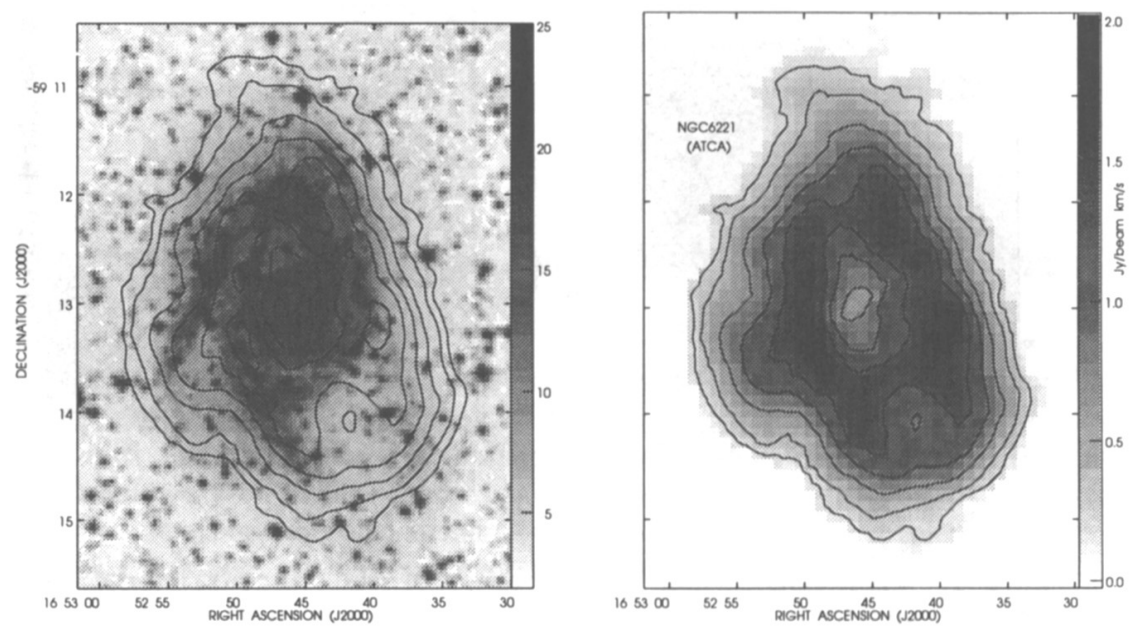

Figure 2. H I distribution (contours) of NGC 6221. The left picture is overlaid onto the optical emission (greyscale) from the DSS. These data were obtained with the $1.5-\mathrm{km}$ array of the ATCA $(12-\mathrm{h})$. The angular resolution is $30^{\prime \prime} \times 25^{\prime \prime}$.

\section{NGC 6221 and Neighbor Galaxies}

NGC 6221 is a barred spiral galaxy which is interacting with its brightest neighbor NGC 6215 and possibly also with two newly discovered low-surface brightness galaxies nearby (one of them is shown in Figure 3). Recent measurements with the ATCA 375-m array revealed an H I bridge between the two big galaxies (Koribalski et al., in prep.). Detailed studies of the $\mathrm{H} \alpha$ emission line in NGC 6221 by Pence \& Blackman (1984) revealed very large non-circular motions of the ionized gas, possibly due to the tidal interaction with NGC 6215 and streaming motions along the bar. The $\mathrm{H}$ I distribution of $\mathrm{NGC} 6221$ is quite similar to the optical emission (see Figure 2). The central depression is due to $\mathrm{H}$ I absorption which is observed over several hundred $\mathrm{km} \mathrm{s}^{-1}$.

\section{NGC 7582 and Neighbor Galaxies}

NGC 7582 is a barred spiral galaxy which is interacting with several bright neighbors, namely NGC 7590, NGC 7599, and another prominent starburst galaxy NGC 7552. Recent measurements with the $375-\mathrm{m}$ array of the ATCA revealed several $\mathrm{H}$ I bridges between the group members. Both NGC 7582 and NGC 7552 reveal broad $\mathrm{H}$ I absorption lines.

The $\mathrm{H}$ I distribution of NGC 7582 (Figure 4) is rather asymmetric (due to the tidal interaction) and extends over a velocity range of at least $400 \mathrm{~km} \mathrm{~s}^{-1}$. The absorption lines cover nearly the same range, but are $\sim 50 \mathrm{~km} \mathrm{~s}^{-1}$ blueshifted with respect to the emission. We clearly detect a shift of the $\mathrm{H} \mathrm{I}$ absorp- 

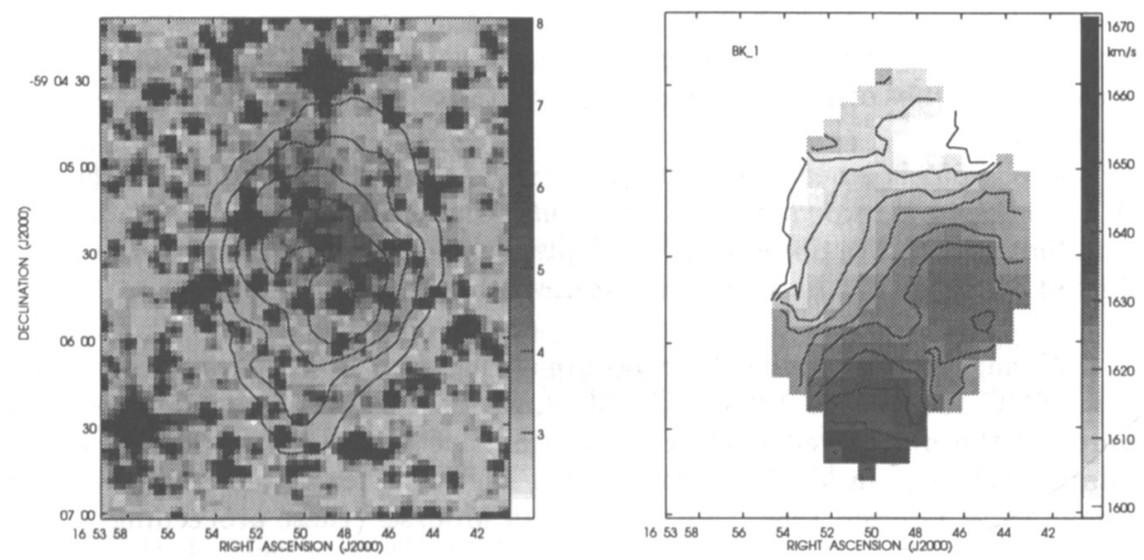

Figure 3. H I distribution (contours) of a newly discovered lowsurface brightness galaxy, BK_1, a companion of NGC 6221, overlaid onto the optical emission (greyscale) from the DSS. The contour levels are $0.1,0.2,0.3,0.4$, and $0.5 \mathrm{Jy}_{\text {beam }}{ }^{-1} \mathrm{~km} \mathrm{~s}^{-1}$ (same data as Figure 2). The mean $\mathrm{HI}$ velocity field is displayed on the right.
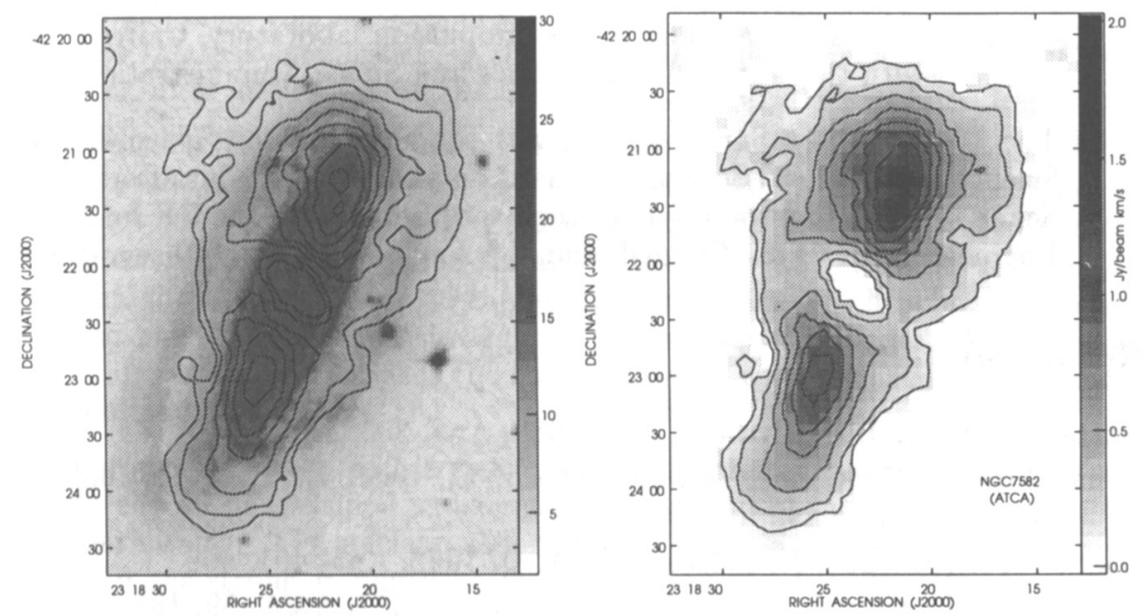

Figure 4. H I distribution (contours) of NGC 7582. The left picture is overlaid onto the optical emission (greyscale) from the DSS. These data were also obtained with the $1.5-\mathrm{km}$ array of the ATCA $(12-\mathrm{h})$. The angular resolution is about $40^{\prime \prime} \times 20^{\prime \prime}$. 
tion line against the continuum emission indicating a fast-rotating ring. Circumnuclear $\mathrm{H} \alpha$ emission suggesting a similar feature (Morris et al. 1985) indicates a mixture of gas phases in the nuclear ring. They also find blue-shifted $O$ III line emission possibly originating from high excitation gas expanding outwards from the nucleus.

NGC 7552 is a nearly face-on $\left(i \approx 28^{\circ}\right)$ galaxy with a strong bar. Forbes et al. (1994) find a $1 \mathrm{kpc}$ starburst ring consisting of supernova remnants and optical 'hot spots'. We observe $\mathrm{H}$ I absorption against the ring and find a velocity shift of $\sim 120 \mathrm{~km} \mathrm{~s}^{-1}$ between its eastern and western part.

We suggest that similar $\mathrm{H}$ I rings are also present in numerous other galaxies (e.g. NGC 1068, NGC 1365, NGC 3079, M 82, NGC 4945, etc.) for which the width of the unresolved nuclear absorption line matches very closely the disk rotation velocity. In M 82 the nuclear ring has been clearly resolved (Weliachew, Fomalont, \& Greisen 1984). Jörsäter \& van Moorsel (these proceedings) discuss NGC 1365.

\section{Acknowledgments.}

- Thanks a lot to my collaborators in the project: John Whiteoak, John Dickey, and Tracy Lavezzi. And I wish to thank especially the numerous students (Sarah Maddison, Cheryl Frost, Brett Hennig, Sally Houghton, and Kate Brooks) who helped during various stages of the observations and data reduction.

- This research has made use of the NASA/IPAC Extragalactic Database (NED) which is operated by the jet propulsion laboratory, Caltech, under contract with the National Aeronautics and Space Administration.

- The Digitized Sky Survey (DSS) was produced by the Space Telescope Science Institute (STScI) and is based on photographic data from the UK Schmidt Telescope, the Royal Observatory Edinburgh, the UK Science and Engineering Research Council, and the Anglo-Australian Observatory.

\section{References}

Antonucci, R. R. J. \& Ulvestad, J. S. 1988, ApJ, 330, L97

Arnaboldi, M., Capaccioli, M., Cappellaro, E., Held, E. V., \& Koribalski, B. 1995, AJ, 110, 199

Baan, W. A., Rhoads, J., \& Haschick, A. D. 1992, ApJ, 401, 508

Combes, F., Gottesman, S. T., \& Weliachew, L. 1977, A\&A, 59, 181

Combes, F. 1988, in Galactic and Extragalactic Star Formation, R. F. Pudritz \& M. Fich, Dordrecht: Kluwer, 475

Combes, F., Braine, J., Casoli, F., Gerin, M., \& van Driel, W. 1992, A\&A, 259, L65

Dickey, J. M., Brinks, E., \& Puche, D. 1992, ApJ, 385, 501

Forbes, D. A. \& DePoy, D. L. 1992, A\&A, 259, 97 
Forbes, D. A., Ward, M. J., Rotaciuc, V., Blietz, M. et al. 1993, ApJ, 406, L11

Forbes, D. A., Norris, R. P., Williger, G. M., \& Smith, R. C. 1994, AJ, 107, 984

Gottesman, S. T. \& Mahon, M. E. 1990, in Paired and Interacting Galaxies, J. Sulentic, W. Keel, \& C. Telesco, Washington, NASA, 209

Koornneef, J. 1993, ApJ, 403, 581

Koribalski, B. \& Dettmar, R.-J. 1993, The ESO Messenger, 71, 37

Koribalski, B., Dickey, J. M., \& Mebold, U. 1993, ApJ, 402, L41

Koribalski, B. 1993, PhD Thesis, University of Bonn

Koribalski, B., Whiteoak, J., \& Houghton, S. 1995, Publ. ASA, 12, 20

Morris, S., Ward, M., Whittle, M., Wilson, A. S., \& Taylor, K. 1985, MNRAS, 216,193

Pence, W. D. 1980, ApJ, 239, 54

Pence, W. D. 1981, ApJ, 247, 473

Pence, W. D. \& Blackman, C. P. 1984, MNRAS, 207, 9

Phillips, A. C. 1993, AJ, 105, 486

Scoville, N. Z., Soifer, B. T., Neugebauer, G., Young, J. S., et al 1985, ApJ, 289, 129

Weliachew, L., Fomalont, E. B., \& Greisen, E. W. 1984, A\&A, 286, 144

\section{Discussion}

W. van Driel: NGC 660 is a peculiar polar ring galaxy; in the NIR it shows a small bar and inner ring features in the principal disk: the $\mathrm{H}$ I absorption feature belongs to the disk kinematics - the polar ring follows a completely different kinematics. It is different from the other SB's in your sample, though.

B. Koribalski: NGC 660 is most likely a polar-ring galaxy, although this is still discussed in the literature (see e.g. Combes et al. 1992; Baan, Rhoads, \& Haschick 1992). It is the only galaxy of this type in the presented sample. But because of its bar and broad H I absorption line (see Gottesman \& Mahon 1990 ) it might well be related to the other barred galaxies. Maybe the bar and polar ring have been created through a merging of two galaxies. And the bar, as usual, drives the gas towards the nuclear region, where it accumulates at the inner Lindblad resonance(s).

T. Hawarden: (1) NGC 660 is clearly a post-merger polar ring: I'm not convinced it should be classified as barred at all (though it may have a ring in the radio continuum). The nuclear absorption in $\mathrm{HI}$ is asymmetric, I think. (2) NGC 3079 has strong $\mathrm{H}_{2}$ emission with the same width as the $\mathrm{H}$ I absorption. (3) NGC 1511 is clearly a post interaction/merger galaxy on the sky surveys.

B. Koribalski: (1) If NGC 660 is not barred, which is difficult to decide, then another mechanism is needed to fuel the nuclear region. Strong tidal interactions between galaxies or galaxy merging are known to do the job (e.g. in Arp 220, NGC 2623, NGC 3690, NGC 6240) and might be a possibility here. (2) That's also the case in NGC 4945, where Koornneef (1993) observed excited $\mathrm{H}_{2}$ emission at $2.2 \mu \mathrm{m}$ with rotational velocities of $200-250 \mathrm{~km} \mathrm{~s}^{-1}$ at a radius of $100 \mathrm{pc}$. (3) 

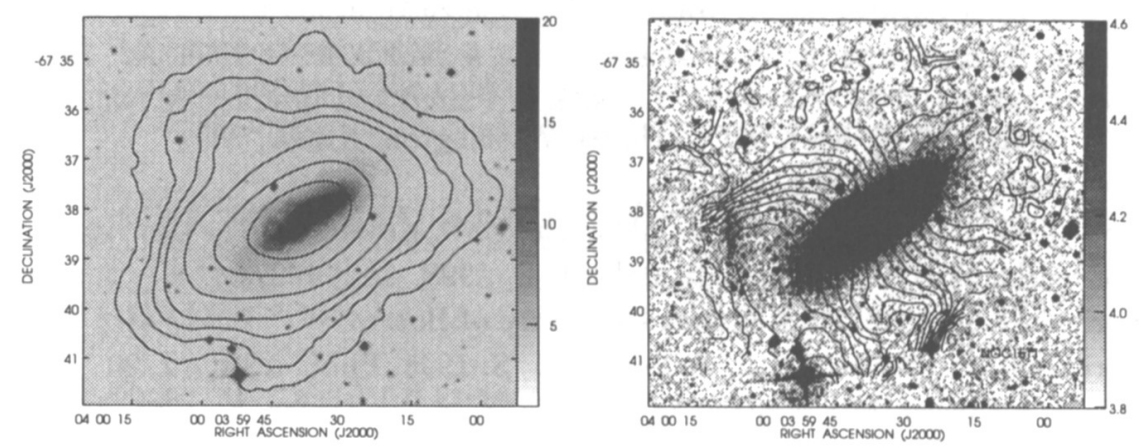

Figure 5. H I distribution and mean velocity field (contours) of NGC 1511 overlaid onto the optical emission (greyscale) from the DSS. The contour levels are $0.3,0.6,0.9,1.2,2.25,4.5$, and $7.5 \mathrm{Jy}^{\text {beam }}{ }^{-1}$ $\mathrm{km} \mathrm{s}^{-1}$ and 1210 to $1435 \mathrm{~km} \mathrm{~s}^{-1}$, step $15 \mathrm{~km} \mathrm{~s}^{-1}$. These data were taken with the $375-\mathrm{m}$ array of the ATCA (12-h). The angular resolution is about $75^{\prime \prime}$.

I have included Figure 5 especially for you. The tidal tail seen in the optical emission (towards the east) is also present in $\mathrm{H} \mathrm{I}$.

Z. Tsvetanov: Given the large velocity amplitude you see in $\mathrm{HI}$ absorption much larger than the $\mathrm{H}$ I emission velocity amplitude - can you say how much more mass in the central regions you would infer from the $\mathrm{H}$ I absorption data vs. the H I emission? Could it be compared with the mass inferred by the stellar velocity curve?

B. Koribalski: The velocity amplitude seen in $\mathrm{H}$ I absorption is in most cases very similar (and not larger) than the $\mathrm{HI}$ emission amplitude. But the absorption is observed at a higher angular resolution and therefore traces gas closer to the nucleus than the emission. The stellar velocity curves, when observed, are similar, but often lack the necessary velocity resolution.

B. Elmegreen: Can you determine how much of the velocity width of the broad absorption lines are from turbulence?

B. Koribalski: With the current synthesis telescopes an angular resolution of a few arcseconds is achieved for 21-cm observations. This is just enough to resolve the nuclear continuum emission in rather nearby galaxies, which enables us to see the rotation of the gas. Much higher resolution is needed to determine the width of the lines caused by turbulence. Although it might be possible for the closest starburst galaxies using the VLA. 